\title{
Neoadjuvant versus adjuvant chemotherapy for resectable non-small cell lung cancer debate revisited
}

\author{
Chan Yeu Pu${ }^{1}$, Sai Yendamuri ${ }^{2,3}$ \\ ${ }^{1}$ Pulmonary, Critical Care and Sleep Division, Department of Medicine, Jacobs School of Medicine and Biomedical Sciences, State University of \\ New York at Buffalo, NY, USA; ${ }^{2}$ Department of Thoracic Surgery, Roswell Park Cancer Institute, Buffalo, NY, USA; ${ }^{3}$ Department of Surgery, Jacobs \\ School of Medicine and Biomedical Sciences, State University of New York at Buffalo, Buffalo, NY, USA \\ Correspondence to: Sai Yendamuri, MD, FACS. Professor and Chair of Thoracic Surgery, Roswell Park Cancer Institute, Buffalo, NY 14263, USA. \\ Email: sai.yendamuri@roswellpark.org. \\ Provenance: This is an invited article commissioned by the Academic Editor Dr. Shuangjiang Li (Department of Thoracic Surgery and West China \\ Medical Center, West China Hospital, Sichuan University, Chengdu, China). \\ Comment on: Brandt WS, Yan W, Zhou J, et al. Outcomes after neoadjuvant or adjuvant chemotherapy for cT2-4N0-1 non-small cell lung cancer: A \\ propensity-matched analysis. J Thorac Cardiovasc Surg 2019;157:743-753.e3.
}

Submitted Oct 30, 2019. Accepted for publication Nov 05, 2019.

doi: $10.21037 /$ jtd.2019.11.23

View this article at: http://dx.doi.org/10.21037/jtd.2019.11.23

The use of adjuvant platinum-based chemotherapy for surgically resectable non-small cell lung cancer (NSCLC) has been well established by several randomized trials and meta-analysis which demonstrated clear survival benefit over no chemotherapy (1-5). However, evidence is less robust regarding the benefit of neoadjuvant chemotherapy (NC). Goals of administering chemotherapy in the preoperative setting (neoadjuvant) include increasing operability by possibly reducing tumor size, improving the likelihood of receiving the maximal planned dose, and reducing the likelihood of micrometastasis. Potential pitfalls of NC include delaying surgery, unnecessary preoperative toxicity affecting postoperative morbidity, and rendering the tumor unresectable (6). A meta-analysis of NC plus surgery versus surgery alone showed significant benefit on survival (7). The National Comprehensive Cancer Network (NCCN) guideline recommends administration of adjuvant chemotherapy (AC) following surgery for T24N0-1 NCSLC but do acknowledge the use of NC instead. The study by Brandt et al. serves to evaluate whether administration of $\mathrm{NC}$ or AC is better for patients with T24N0-1 NCSLC undergoing surgical resection (8).

Brandt et al. performed a propensity score match analysis of a single institution patients with T2-4N0-1 NCSLC who underwent surgical resection and compared outcomes after $\mathrm{NC}$ versus $\mathrm{AC}$. The $\mathrm{NC}$ and $\mathrm{AC}$ groups were matched for age, year of surgery, gender, body mass index (BMI), Charlson comorbidity score, type of surgery (pneumonectomy, bilobectomy, lobectomy, or segmentectomy), tumor laterality, histology subtype (adenocarcinoma, squamous, large cell, mixed, other), clinical stage (IB, IIA, IIB, IIIA) and pretreatment tumor $\mathrm{SUV}_{\text {max }}$ which created a balanced group of 92 patients each. The 5-year overall survival (OS) was 64\% (95\% CI: $54-$ $75 \%)$ for $\mathrm{NC}$ and $61 \%$ for AC (95\% CI: $50-74)$ which were not significantly different on univariate and multivariate analysis HR 1.21 (95\% CI: 0.63-2.30, P=0.57). Several previous investigators have tried to answer this question. For example, the three arm NATCH trial compared NC plus surgery versus AC plus surgery versus surgery alone and found no benefit of NC nor AC to surgery, the study had a number of limitations (9). The NATCH trial was criticized for being overly optimistic with a power analysis of $15 \%$ difference in disease-free survival (DFS) at 5 years and over representation by stage I disease patients (75\%). Since there are limited head to head trials comparing $\mathrm{NC}$ to AC, the meta-analysis of $\mathrm{NC}$ versus AC by Lim et al. also did not show difference in overall and DFS between NC and AC (10).

The argument for using chemotherapy before rather than after surgery largely comes from a difference in tolerability of chemotherapy that was supported by the NATCH 
phase 3 trial which showed that $90 \%$ of the preoperative cohort completed 3 cycles of chemotherapy compared to only $61 \%$ in the postoperative cohort (9). Consistent with this prior study, the $\mathrm{NC}$ group in Brandt et al. were more likely to complete chemotherapy compared to AC group (86 versus 72 patients). Nonetheless, improvement in the number of chemotherapy cycles delivered did not result in improvement in OS in other studies $(9,11)$ and in the study by Brandt et al.

The concern of NC is the theoretical worsening of surgical outcome due to toxicity. In Brandt et al.'s study, the severe postoperative complication rate in NC was $14 \%$ compared to $7 \%$ in AC group but was not significantly different. The relatively small number of patients in each group may have limited the detection of significance. In comparison, with 624 patients, the peri-operative mortality in the NATCH trial was similar between NC plus surgery versus AC plus surgery versus surgery alone groups (9).

The use of NC has been shown to have a greater potential to eradicate micro-metastasis leading to $10 \%$ absolute benefit on distant recurrence at 5 years (7). Since $\mathrm{NC}$ has greater effect on preventing metastasis and AC better for local control (4), the combination of NC and AC may confer greater benefit on local and distant control leading to better survival. The open label randomized trial by Westeel et al. did not show survival difference in earlystage NSCLC patients receiving 4 cycles of preoperative chemotherapy compared to patients receiving 2 cycles preoperatively followed by 2 cycles postoperatively (11). Unfortunately, the inclusion of patients with stage 1A $(17.4 \%)$ and $1 \mathrm{~B}(47 \%)$ in which the benefit of combining chemotherapy with surgery is less clear, may have affected the study outcome.

The author showed that NC might provide additional prognostic information based on chemotherapy response of the resected tumor. Major pathologic response (MPR) is defined as $10 \%$ or less residual viable tumor after NC (12). Kaplan-Meier curves of MPR indicated better OS and DFS versus no MPR in the NC group. And the tumor Response Evaluation Criteria in Solid Tumor (RECIST) response to $\mathrm{NC}$ was significantly associated with improve DFS. This indicates their potential use as surrogate measurement of survival, available by the use of NC. The inclusion of stage IB NCSLC patients in this study which comprise $10 \%$ and $12 \%$ of neoadjuvant and adjuvant groups respectively may have affected the results because the evidence for use of chemotherapy in this group is less established than in stage II and III. Although stage 1B patients was included in the landmark AC trials (1-5), the CALGB 9633 which only studied AC in stage $1 \mathrm{~B}$ patients did not show survival advantage (13). Nevertheless, the study maybe underpowered (14). The NCCN guidelines only recommends AC in stage 1B NCSLC with negative margin if the patient is high risk. Definition of high risk includes poor differentiated tumors, vascular invasion and visceral pleural involvement. Hence, a study comparing NC versus AC in resectable NSCLC should focus on stage II-III (N1 disease) where the indication for chemotherapy is clear but the timing is not clear, rather than on stage $1 \mathrm{~A}$ or $1 \mathrm{~B}$ (N0 disease).

A general limitation of all propensity score match study is that if a crucial variable is omitted, the groups will be unbalanced leading to bias. This is especially true in retrospective studies that use a preexisting database which is limited to the variables collected rather than having all the variables needed to make the groups balanced (15). In this study, although the risk from surgery was accounted for by the including the type of surgery into the propensity score, the pulmonary function tests measurements such as forced expiratory volume in 1 second $\left(\mathrm{FEV}_{1}\right)$, forced vital capacity (FVC) and diffusing capacity of the lung for carbon monoxide (DLCO) were not included. Post pneumonectomy AC was found to improve OS in patients with preoperative $\mathrm{FEV}_{1}$ of more than $2 \mathrm{~L}$ but has no effect on patients with preoperative $\mathrm{FEV}_{1}$ of less than $2 \mathrm{~L}$ (16). Another important variable to consider in the propensity score matching is the surgical margin which was also not included in this study. The process of propensity score matches the patients in the middle where their score overlaps. However, these patients might not be representative of their parent groups. The patient may be the upper extreme of one group and the other patient is of the lower extreme of the other group (15). The patients in NC group were more likely to have earlier year surgery, pneumonectomy, higher clinical stage, higher pretreatment $\mathrm{SUV}_{\text {max }}$ and adenocarcinoma than the AC group before propensity score matching. By make the groups similar, the outcome showing no difference in OS between NC and AC might be because the two matched groups are very similar but is not representative of their actual unmatched patient cohort, which makes the findings not generalizable.

While this study tries to tackle a problem that surgeons have been wrestling with for a long time, it may soon be irrelevant. There is a distinct movement within thoracic oncologists toward NC. Neoadjuvant immunotherapy and chemoimmunotherapy is logical as it takes advantage of the presence of the tumor to generate an immune response 
before its removal. If this becomes the standard of care for chemotherapy, then the current debate of timing may become irrelevant. For the present, the best timing for chemotherapy in resectable N1 NSCLS is unclear in view of the different benefits and drawbacks associated with each approach. At this point, the decision should be personalized to the treatment team and the patients while waiting for additional prospective studies to answer the optimal timing of chemotherapy in surgically resectable N1 NSCLC patients.

\section{Acknowledgments}

None.

\section{Footnote}

Conflicts of Interest: The authors have no conflicts of interest to declare.

Etbical Statement: The authors are accountable for all aspects of the work in ensuring that questions related to the accuracy or integrity of any part of the work are appropriately investigated and resolved.

\section{References}

1. Pignon JP, Tribodet H, Scagliotti GV, et al. Lung adjuvant cisplatin evaluation: a pooled analysis by the LACE Collaborative Group. J Clin Oncol 2008;26:3552-9.

2. Arriagada R, Bergman B, Dunant A, et al. Cisplatinbased adjuvant chemotherapy in patients with completely resected non-small-cell lung cancer. $\mathrm{N}$ Engl J Med 2004;350:351-60.

3. Winton T, Livingston R, Johnson D, et al. Vinorelbine plus cisplatin vs. observation in resected non-small-cell lung cancer. N Engl J Med 2005;352:2589-97.

4. NSCLC Meta-analyses Collaborative Group, Arriagada R, Auperin A, et al. Adjuvant chemotherapy, with or without postoperative radiotherapy, in operable non-small-cell lung cancer: two meta-analyses of individual patient data. Lancet 2010;375:1267-77

5. Douillard JY, Rosell R, De Lena M, et al. Adjuvant vinorelbine plus cisplatin versus observation in patients with completely resected stage IB-IIIA non-small-cell lung cancer (Adjuvant Navelbine International Trialist Association [ANITA]): a randomised controlled trial. Lancet Oncol 2006;7:719-27. Erratum in: Lancet Oncol 2006;7:797.

6. McElnay P, Lim E. Adjuvant or neoadjuvant chemotherapy for NSCLC. J Thorac Dis 2014;6: S224-7.

7. NSCLC Meta-analysis Collaborative Group. Preoperative chemotherapy for non-small-cell lung cancer: a systematic review and meta-analysis of individual participant data. Lancet 2014;383:1561-71.

8. Brandt WS, Yan W, Zhou J, et al. Outcomes after neoadjuvant or adjuvant chemotherapy for cT2-4N0-1 non-small cell lung cancer: A propensity-matched analysis. J Thorac Cardiovasc Surg 2019;157:743-753.e3.

9. Felip E, Rosell R, Maestre JA, et al. Preoperative chemotherapy plus surgery versus surgery plus adjuvant chemotherapy versus surgery alone in early-stage nonsmall-cell lung cancer. J Clin Oncol 2010;28:3138-45.

10. Lim E, Harris G, Patel A, et al. Preoperative versus postoperative chemotherapy in patients with resectable non-small cell lung cancer: systematic review and indirect comparison meta-analysis of randomized trials. J Thorac Oncol 2009;4:1380-8.

11. Westeel V, Quoix E, Puyraveau M, et al. A randomised trial comparing preoperative to perioperative chemotherapy in early-stage non-small-cell lung cancer (IFCT 0002 trial). Eur J Cancer 2013;49:2654-64.

12. Hellmann MD, Chaft JE, William WN Jr, et al. Pathological response after neoadjuvant chemotherapy in resectable non-small-cell lung cancers: proposal for the use of major pathological response as a surrogate endpoint. Lancet Oncol 2014;15:e42-50.

13. Strauss GM, Herndon JE 2nd, Maddaus MA, et al. Adjuvant paclitaxel plus carboplatin compared with observation in stage IB non-small-cell lung cancer: CALGB 9633 with the Cancer and Leukemia Group B, Radiation Therapy Oncology Group, and North Central Cancer Treatment Group Study Groups. J Clin Oncol 2008;26:5043-51.

14. Katz A, Saad ED. CALGB 9633: an underpowered trial with a methodologically questionable conclusion. J Clin Oncol 2009;27:2300-1; author reply 2301-2.

15. Streiner DL, Norman GR. The pros and cons of propensity scores. Chest 2012;142:1380-2.

16. Wang $M$, Zhao J, Su YJ, et al. Role of adjuvant chemotherapy after pneumonectomy for non-small cell lung cancer. Oncol Lett 2012;4:1349-53.

Cite this article as: $\mathrm{Pu} \mathrm{CY}$, Yendamuri S. Neoadjuvant versus adjuvant chemotherapy for resectable non-small cell lung cancer debate revisited. J Thorac Dis 2019;11(12):5646-5648. doi: $10.21037 /$ jtd.2019.11.23 